\title{
Kussmaul breathing
}

National Diabetes Information Clearinghouse (NDIC)

\section{Definitions}

Diabetic ketoacidosis (DKA)

Defined by National Diabetes Information Clearinghouse (NDIC)

\section{Source}

National Diabetes Information Clearinghouse (U.S.). (2009). The diabetes dictionary. [Bethesda, Md.]: U.S. Dept. of Health and Human Services, National Institutes of Health, National Institute of Diabetes and Digestive and Kidney Diseases, National Diabetes Information Clearinghouse.

The rapid, deep, and labored breathing of people who have diabetic ketoacidosis. 